\title{
On Hybrid Wave Functions, Tidal Forces and Black Holes
}

\author{
Leon Francis Phillips \\ Department of Chemistry, University of Canterbury, Christchurch, New Zealand
}

\section{Email address:}

leon.phillips@canterbury.ac.nz

\section{To cite this article:}

Leon Francis Phillips. On Hybrid Wave Functions, Tidal Forces and Black Holes. American Journal of Astronomy and Astrophysics. Vol. 5, No. 1, 2017, pp. 6-9. doi: 10.11648/j.ajaa.20170501.12

Received: January 22, 2017; Accepted: February 3, 2017; Published: March 2, 2017

\begin{abstract}
Interesting questions that arise in connection with the physics of black holes include the existence or nonexistence of mathematical singularities in the physical universe, the rate at which an incoming particle can be observed to cross a black hole's event horizon, the quantum mechanics of small particles inside a gravitational potential well, the rate of absorption of an incoming particle into the core of a black hole, the mechanism by which very high-energy particles can be emitted from a black hole's potential well, and the nature of the interface between quantum mechanics and general relativity. The purpose of this Note is to suggest plausible answers to some of these questions. The discussion focuses on basic physics rather than mathematics, and quantum mechanics and general relativity are regarded as equally fundamental.
\end{abstract}

Keywords: No Singularities, Tidal Forces, Hybrid Wave Functions, Black Hole Core, High-Energy Particle Emission

\section{Introduction: On Singularities}

A black hole is generally agreed to be the product of catastrophic gravitational collapse of a massive star, and the elegant mathematics of this process has often been interpreted as implying that it gives rise to a mathematical singularity at the core of the black hole [1]. However this interpretation is untenable for a number of reasons [2], of which the most fundamental is that Einstein's General Relativity provides the large-scale arena in which the geometry and physics of the universe function but the physics of the universe on a very small scale is governed by quantum mechanics, and the existence of a point-like mathematical singularity in the physical universe is ruled out by the thoroughly-tested Heisenberg uncertainty principle.

Other considerations include the circumstance that disappearance of incoming particles into a mathematical singularity provides no clear avenue for growth of a black hole via capture of incoming mass-energy, despite the obvious existence of super-massive black holes, and even super-massive binary black holes, in the universe [3]. The solutions of Einstein's General Relativity equations that give rise to a singularity at or near the location of a black hole's ultra-dense core clearly are correct for empty space-time. However, the space-time of the current universe is not empty, the core of a black hole has a non-zero radius, and the very beautiful mathematical solutions have not been proven to apply inside the spheroidal, quasi-solid core of the black hole where the singularity is supposed to reside. The assumption that incoming mass-energy readily disappears into a singularity leads to the conclusion that the temperature at the surface of the core of a medium-sized black hole is at most a few Kelvin [4], which is unreasonable in view of the great depth of the gravitational potential well that an incoming particle has to traverse, acquiring enormous kinetic energy along the way, before it has the opportunity to merge with the core [5]. Hence, rather than being very cold, the surface of a black hole's core is more likely to consist of a quark-gluon plasma [6] with a temperature in the region of $10^{12}$ Kelvin.

\section{Rates of Crossing the Event Horizon by a Particle, and of Particle Absorption Into a Black Hole Core}

It is often stated in textbooks that, from the viewpoint of an external observer, the rate of crossing a black hole's event horizon by an incoming particle is infinitely slow. This statement might well be correct for a particle of negligible mass, but it cannot hold for a particle whose mass is sufficient to distort the geometry of space-time in the vicinity of the crossing point. Thus a recent study [7] reports the tidal disruption of a star that evidently approached too close for 
comfort to a black hole, the disruption beginning to occur at a separation greater than the radius of the event horizon, and a significant amount of the mass-energy of the disrupted star being ejected in the form of a jet extending for several light years in a direction away from the black hole. (Note that, when there is only a single jet, conservation of momentum requires a balancing amount of mass-energy to be directed unseen into the black hole core, which provides a clear avenue for growth of the black hole.) More often, in such systems, there is a pair of jets, leaving above and below the accretion disk, as in the diagram below, but their intensities are seldom equal and any imbalance is transferred to the black hole's core.

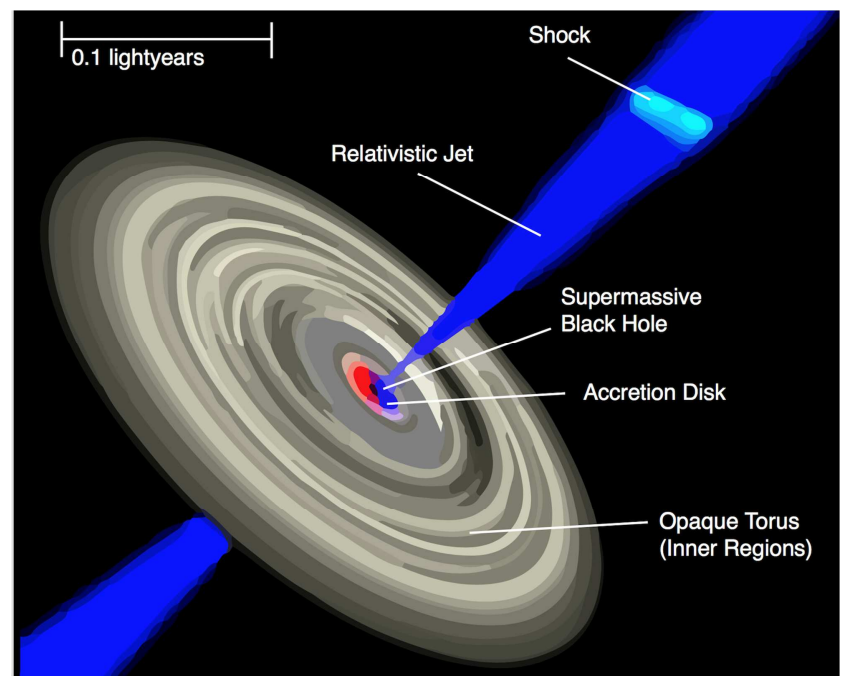

Fig. 1. Black hole with a pair of jets (from Wikipedia).

Tidal disruption of intermediate-sized particles can give rise to similar jet phenomena [8] in the presence of accretion disks that comprise a cloud of small and medium-sized particles generated by the disruption. It is evident that infinitely slow processes can have very little to do with the growth of black holes.

After an incoming particle has penetrated the event horizon of a black hole, its absorption into the ultra-dense core is necessarily quite a slow process, because the wave functions of particles captured by a black hole's gravitational potential well almost invariably correspond to states with non-zero angular momentum, and such wave functions have zero amplitude at the origin [9]. Hence a captured particle must spend a significant amount of time within the system of discrete energy levels that quantum mechanics requires to exist in the potential well, before eventually being absorbed into the core. This implies the possibility that the energy levels might eventually fill with particles, mainly in the form of captured atomic and sub-atomic fragments, up to and beyond the event horizon.

The radius of a black hole's quasi-solid core is many orders of magnitude smaller than the radius of the event horizon, so the mathematical origin of the wave function of a captured particle with non-zero angular momentum must be very close to the core's surface. In order for the core of the black hole to grow, the captured particle must perturb the curvature of space-time in the vicinity of the core to such an extent that its wave function does not go precisely to zero at the surface, but is able to tunnel a short distance into the core. Then, instead of simply bouncing off the core like a particle in a box, the particle has a small but definite probability of being absorbed.

The volume of a black hole's massive core is so much smaller than the volume of the portion of the potential well that is inside the event horizon but outside the core, that captured-but-not-yet-absorbed fermions within the black hole's potential well must be distributed over the available hydrogen-like energy levels [5] in accordance with the Pauli principle. After a significant amount of time has elapsed since the black hole's formation, the thermal distribution of neutrinos and other small particles over the available energy levels might result in the population of levels up to and beyond the event horizon. Such a process could then, as a result of collisions which sent one colliding particle of an entangled pair plunging into the depths of the potential well and the other flying off into the surrounding space-time, provide a source of high-energy neutrinos such as those observed by the Ice Cube collaboration [10]. Also, because the surface temperature of a black hole's core is very high, other particles, even including weakly interacting bosons, can reasonably be expected to distribute themselves over the large number of discrete energy levels available in a deep gravitational well (typically $\sim 10^{60}$ levels for a neutrino, $\sim 10^{25}$ for an electron, and $\sim 10^{10}$ for a neutron, for a black hole of $\sim 10^{6}$ solar masses [11]). The corresponding wave functions might leak out through the event horizon and give rise to energetic particles that could be detected by an external observer.

\section{The Role of Hybrid Wave Functions}

The wave functions of particles located between the surface of the black hole core and the event horizon are not easy to describe because the system is not static. However, a useful clue is provided by the fact that three space-like coordinates and one time-like coordinate are available, and the use of a linear combination of all four coordinates is compulsory for describing the behavior of the fourdimensional object that is the wave function of a small particle. Large particles captured by the potential well are less easily characterized in terms of wave functions than small ones, but this is not a serious problem because every large particle must eventually be broken into very small particles by tidal forces, and the motion of the resulting subatomic and sub-nuclear particles in the region inside the event horizon is governed by quantum mechanics. This suggests an interesting topic for future study, namely, the quantum mechanics of systems comprised of several different types of small particles within the gravitational potential well of a spinning black hole.

Thus the four-dimensional wave functions of small particles inside the gravitational potential well are expected 
to be hybrids of one time-like and three space-like functions. This situation is reminiscent of the $s p^{3}$ hybridization of electron orbitals that is a feature of the quantum mechanics of small polyatomic molecules, with the difference that here the form of the hybrid wave functions is strongly dependent on the extent of deviation of the black hole from spherical symmetry. Also there are interesting complications that arise from both the great depth of the gravitational potential well and the simultaneous presence of a variety of different particles. The wave functions of small particles in the energy levels nearest to the black hole's core must be able to tunnel into the core, but they are likely to be greatly attenuated in the process, the rate of attenuation being greater for a denser core. Hence the rate of absorption of incoming mass-energy into the core could well differ significantly between relatively light, newly-formed black holes and very massive old ones.

\section{The Interface Between Quantum Mechanics and General Relativity}

The idea that the value $c$ of the speed of light in a vacuum represents an upper limit to the attainable speed for a moving particle or photon is one of the unchallenged foundations of Einstein's Special Relativity. However, there is still scope for time-variation of the value of the maximum attainable speed, as proposed by Guth [12] and expounded very clearly by Magueijo [13]. Such variation is not surprising, because the speed of light in a vacuum is fixed by the permittivity and permeability of empty space, and these properties, which relate to the transient production of virtual particles in the vacuum, can be expected to change quite dramatically as the available volume of vacuum varies from near-zero up to the present size of the universe.

It was pointed out by Hughes more than a decade ago [14], that there is a loophole in J. A. Wheeler's 'black holes have no hair' statement, to the effect that it applies quite rigorously to a passive Schwarzschild black hole but much less rigorously to a Kerr black hole that is both spinning on its axis and evolving dynamically by capturing material from an accretion disk. Hence elementary particles should be able to escape from a non-stationary black hole at a much greater rate than is predicted for Hawking radiation. In general it appears likely that both capture and escape should occur most efficiently along the axis of rotation, and such escape processes might contribute to interesting observational phenomena, such as the energetic jets that have been observed to extend for several light-years from their blackhole source [8]. As noted above, for an entangled pair of fermions, this could provide an energy-pooling mechanism that would allow one particle of the pair to escape to infinity while the other was falling into the depths of the gravitational potential well.

The simplest description of the interface between general relativity and quantum mechanics is that general relativity controls the large-scale geometry of the universe and quantum mechanics governs small-scale phenomena. In situations where the magnitudes of large-scale and smallscale phenomena overlap, general relativity provides the blank surface of space-time upon which quantum mechanics writes the fine print.

\section{Conclusions}

For the reasons outlined in the introduction, mathematical singularities cannot exist in the physical universe, and the speed at which a massive incoming particle can be observed to cross the event horizon of a black hole is necessarily greater than zero. The surface of a black hole's core is strongly heated by the impacts of incoming particles. The momentum of jets of mass-energy emitted outwards from an accretion disk is approximately balanced by the momentum of invisible jets emitted inwards towards the black hole core, this providing a clear mechanism for growth of the core. Particles captured inside the event horizon occupy discrete energy levels in the gravitational potential well. The wave functions of such particles are hybrids of one time-like and three space-like functions. Captured particles usually have non-zero angular momentum, as a consequence of which their wave function is zero at the origin and they merge only slowly with the black hole core. Binary collisions involving an entangled pair of particles in high energy levels of a potential well can result in one of the particles being emitted outwards with very large kinetic energy, and the other being emitted invisibly in the direction of the core. Spin is ubiquitous in the universe so, in applications involving black holes, it is essential to work with Kerr rather than Schwarzschild geometry. At the time of writing, and despite much effort by serious theoreticians, no instances have been found that clearly require the existence of an arcane discipline called 'quantum gravity'. However, the universe is a big place, so quantum gravity, like string theory, cannot yet be ruled out.

\section{References}

[1] See, for example Misner, C. W., Thorne, K. S. and Wheeler, J. A., (1973), Gravitation, Freeman, San Francisco, p. 846.

[2] Neves, J. C. S. and Saa, A., (2014) Regular rotating black holes and the weak energy condition, Phys. Lett. B., 734, 4448.

[3] Bon, E., Jovanović, P., Marziani, P., Shapovalova, A. I., Bon, N., Borka, V., Jovanović, D., Borka, D., Sulentic, J., and Popović, L. Č. (2012) The first spectroscopically resolved sub-parsec orbit of a super-massive binary black hole, Astrophys. J, 118, 759.

[4] Hawking, S. W. (1974) Black hole explosions? Nature (London) 248, 30.

[5] Phillips, L. F. (2015) Energy levels of neutrinos in a gravitational potential well, App. Phys. Research, 7 (1), 19.

[6] Wang, S. Y., and Boyanovsky, D. (2001) Enhanced photon production from quark-gluon plasma: Finite-lifetime effect, Phys. Rev. D., 63, 051702 (R). 
[7] Leloudas, G., Fraser, M., Stone, N. C., et al, The superluminous transient ASASSN-15lh as a tidal disruption event from a Kerr black hole, arXiv: 1609.02927v2 [astro-ph. HE] 11 Dec 2016.

[8] Nemmen, R. S., Georganopoulos, M., Guiriec, S., Meyer, E. T., Gehrels, N., Sambruna, R. M. (2012) A universal scaling for the energetics of relativistic jets from black hole systems, Science, 338, 1445.

[9] See for example, Eyring, H., Walter, J., and Kimball, E. M. (1944) Quantum Chemistry, Wiley, New York p. 86.

[10] Aartsen, M. G. et al (the Ice Cube collaboration) (2013) First observation of PeV-energy neutrinos with Ice Cube, Phys. Rev. Lett., 111 (2), 021103.

[11] Phillips, L. F. (2015) Black holes as a source of high-energy neutrinos, App. Phys. Research, 7 (4), 1.

[12] Guth, A. H. (1993) Inflation, Proc. Nat. Acad. Sci. U. S. A., 90 (11), 4871-7.

[13] Magueijo, J. (2003) Faster than the speed of light, Arrow Books, London.

[14] Hughes, S. A. (2005) 33rd SLAC Summer Institute on Particle Physics. 